\title{
The influence of PSA flare in MCRPC patients treated with alpha-emitting radiopharmaceuticals
}

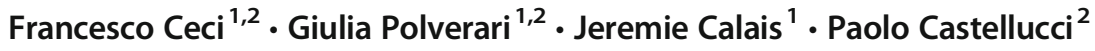 \\ Published online: 19 September 2018 \\ (C) Springer-Verlag GmbH Germany, part of Springer Nature 2018
}

In this issue of the European Journal of Nuclear Medicine, Castello et al. [1] performed a retrospective analysis in 168 metastatic castration-resistant prostate cancer (mCRPC) patients who underwent ${ }^{223} \mathrm{RaCl}_{2}$. All patients presented bone metastases and $34 \%$ of these patients had concomitant visceral and/or nodal metastases. Authors evaluated the prognostic impact of PSA flare on overall survival (OS) and progression-free survival (PFS) in this cohort. This is the first study specifically designed to assess the presence and the influence of PSA flare in patients undergoing alpha-emitting radiopharmaceuticals.

The PSA flare is a well-known phenomenon observed in several studies in the course of systemic therapies, including hormonal therapies (especially luteinizing hormone-releasing hormone (LHRH) agonist), new androgen receptor targeted therapies (abiraterone acetate or enzalutamide) and taxane-based chemotherapy (either docetaxel or cabazitaxel) [2]. A transient PSA rise followed by a decrease of PSA below the baseline can be considered a temporary flare-up. The cause of such flare has only been postulated. Many suggest that it could be caused by PSA release from lysed cells. However, the aberrant activation of the androgen receptor or the transactivation of the androgen receptor induced by corticosteroids have been suggested as the

This Editorial Commentary refers to the article https://oi.org/10.1007/ s00259-018-4051-y

Francesco Ceci

fceci@mednet.ucla.edu

1 Ahmanson Imaging Translational Division, Department of Molecular and Medical Pharmacology, University of California Los Angeles (UCLA), Los Angeles, CA, USA

2 Metropolitan Nuclear Medicine, S.Orsola-Malpighi University Hospital, University of Bologna, Bologna, Italy possible cause responsible for PSA flare-up during systemic therapy [3]. Other explanations are related to cellular heterogeneity and diversity in terms of drug sensitivity, cell cycle kinetics, and PSA expression [4].

The development of PSA flare does not influence diseasespecific outcomes. Its incidence is attested in $5-30 \%$ of patients receiving LHRH agonist and in $8-20 \%$ of patients receiving chemotherapy $[2,5]$. As treatment of mCRPC becomes more complex, and definitions of progression on treatment continue to evolve, referring physicians must be aware of this laboratory pattern to avoid unnecessary discontinuation of these therapies based on early PSA change alone.

Castello et al. [1] confirmed the presence of PSA flare-up also in $\mathrm{MCRPC}$ patients treated with alpha-emitting radiopharmaceuticals $\left({ }^{223} \mathrm{RaCl}_{2}\right)$. Although there is no agreed definition on the extent of the following PSA decline to be considered as a flare after an initial rise in PSA, a flare followed by a decrease below the baseline is generally considered the most accepted definition. According to these criteria, authors observed PSA flare-up in $11.9 \%$ of their population. In the flare group, OS and PFS were considerably better than in the nonresponder group. These results are consistent with data obtained in mCRPC patients undergoing abiraterone or taxanebased chemotherapies $[2,3,5,6]$.

${ }^{233} \mathrm{RaCl}_{2}$ preferentially targets new bone growth surrounding bone metastases while emitting alpha particles within the tumour microenvironment. Whereas beta particles generate primarily single-stranded deoxyribonucleic acid breaks that may be overcome by cellular repair mechanisms, alpha particles have high linear energy transfer with enhanced ability to induce lethal double-stranded deoxyribonucleic acid breaks, thus eliciting greater cytotoxic effects on bone-metastatic tumour sites [7]. Accordingly, Castello et al. hypothesized that PSA flare during ${ }^{233} \mathrm{RaCl}_{2}$ treatment is due to PSA release from tumour cell lysis, and may correspond to a high degree of tumour cell death. However, the concomitant administration of therapies, including hormonal therapy, can be responsible for the PSA flare-up in this patient-series, through the 
activation of the androgen receptor. Nevertheless, regardless of the mechanisms involved, ${ }^{233} \mathrm{RaCl}_{2}$ therapy should not be discontinued after an early and transient PSA rise. The PSA flare induced by alpha-emitting therapy during, at least, the first 2 months of treatment (one to two cycles), does not represent a proven sign of disease progression.

Since the introduction of PSA screening, prostate cancer diagnosis and management have been guided by this biomarker based on the assumption that PSA levels have been shown to be associated with disease burden [8]. However, this correlation becomes more complicated in advanced disease states that are characterized by increasing disease cells heterogeneity, due to the complex mechanisms involved in the development of castration-resistant prostate cancer [9]. Furthermore, recent studies have described the presence of active visceral metastases not producing PSA [10]. For these reasons, the Prostate Cancer Clinical Trial Working Group 3 (PCWG3) suggest also using other blood-based markers (e.g., alkaline phosphatase, lactate dehydrogenase, and circulating tumour cells) to follow up patients treated with systemic treatment [11].

At present, the usefulness of imaging for monitoring the response to therapy in $\mathrm{mCRPC}$ patients is being debated [12]. Bone scan (either sodium-fluoride PET or bone scintigraphy) is widely used in clinical practice, and has been proposed in some studies [13] for predicting the response to bone-targeted therapy, especially alpha-emitting radiopharmaceuticals. However, this imaging modality evaluates only the skeletal involvement by prostate cancer metastases. Thus, the eventual progression of the disease in other sites cannot be assessed. Furthermore, its efficacy is impaired by the bone scan flare phenomenon (the development of new lesions on a first follow-up scan that may actually do not represent disease progression). Recently, highly successful results have been obtained targeting the prostate specific membrane antigen (PSMA), both for diagnostic and therapeutic purposes. PSMA-11 PET/CT outperformed choline PET/CT in patients presenting PSA failure after radical therapy [14], but its role in later stages has not been extensively validated. On the other hand, in advanced stages of the disease the use of PSMAligands as therapeutic agent is gaining importance.

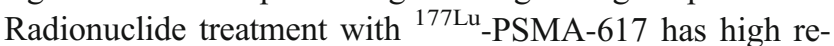
sponse rates, low toxic effects, and reduction of pain in men with $\mathrm{mCRPC}$ who have progressed after conventional treatments [15]. Thus, PSMA may represent the ideal in-vivo biomarker for theranostic purposes in prostate cancer. At present, the incidence of PSA flare in patients treated with PSMAbased radioligand therapy (either with beta or alpha emitters) has not been reported in large patient-series. However, the presence of this phenomenon is largely predictable also in those patients treated with this innovative therapy.

PCWG3 suggest that early PSA rise should be ignored before 12 weeks of treatment in determining PSA response [11]. Accordingly, there is an unmet need to assess the early response to therapy in order to minimize patient exposure to ineffective therapy. The availability of a diagnostic procedure able to assess the response to therapy accurately and at an early stage may have an important impact on mCRPC management. Although PSMA PET/CT cannot completely replace bone scan for detecting active osteoblastic lesions in patients treated with alpha-emitting radiopharmaceuticals, PSMA PET/CT may help with better treatment planning. Patients with undetected nodal or visceral metastases or bone-marrow involvement should be restaged and addressed to the most tailored treatment strategy. In addition to this approach providing an improvement in life expectancy, the collateral effects, toxicity, and costs of futile therapy will be reduced.

In conclusion, the presence of PSA flare has been described also in mCRPC patients treated with ${ }^{223} \mathrm{RaCl}_{2}$ [1]. Considering the heterogeneity of PSA expression in advanced prostate cancer, this biomarker is less reliable in advanced stages of the disease. The role of PSMA PET imaging in this scenario has not been extensively explored, but deserves more attention in the light of the increasing interest of this radiopharmaceuticals as a therapeutic agent.

\section{Compliance with ethical standards}

Ethical approval All procedures performed in studies involving human participants were in accordance with the ethical standards of the institutional and/or national research committee and with the 1964 Helsinki Declaration and its later amendments or comparable ethical standards.

Conflict of interest The authors declare that they have no conflict of interest.

\section{References}

1. Castello A, Macapinlac HA, Lopci E, Santos EB. Prostate-specific antigen flare induced by $(223) \mathrm{RaCl}(2)$ in patients with metastatic castration-resistant prostate cancer. Eur J Nucl Med Mol Imaging. 2018 [Epub ahead of print]. https://doi.org/10.1007/s00259-0184051-y.

2. Nelius T, Filleur S. PSA surge/flare-up in patients with castrationrefractory prostate cancer during the initial phase of chemotherapy. Prostate. 2009;69(16):1802-7.

3. Shiota M, Yokomizo A, Takeuchi A, et al. Co-introduction of a steroid with docetaxel chemotherapy for metastatic castrationresistant prostate cancer affects PSA flare. BJU Int. 2016;118: $880-4$.

4. van der Poel HG, Oosterhof GO, Schaafsma HE, Debruyne FM, Schalken JA. Intratumoral nuclear morphologic heterogeneity in prostate cancer. Urology. 1997;49(4):652-7.

5. Ueda Y, Matsubara N, Tabata KI, Satoh T, Kamiya N, Suzuki H, et al. Prostate-specific antigen flare phenomenon induced by Abiraterone acetate in chemotherapy-naive patients with metastatic castration-resistant prostate cancer. Clin Genitourin Cancer. 2017;15(2):320-5. 
6. Burgio SL, Conteduca V, Rudnas B, Carrozza F, Campadelli E, Bianchi E, et al. PSA flare with abiraterone in patients with metastatic castration-resistant prostate cancer. Clin Genitourin Cancer. 2015;13(1):39-43. https://doi.org/10.1016/j.clgc.2014.06.010.

7. Hoskin P, Sartor O, O'Sullivan JM, Johannessen DC, Helle SI, Logue J, et al. Efficacy and safety of radium-223 dichloride in patients with castration-resistant prostate cancer and symptomatic bone metastases, with or without previous docetaxel use: a prespecified subgroup analysis from the randomised, double-blind, phase 3 ALSYMPCA trial. Lancet Oncol. 2014;15(12):1397-406.

8. Carvalhal GF, Daudi SN, Kan D, Mondo D, Roehl KA, Loeb S, et al. Correlation between serum prostate-specific antigen and cancer volume in prostate glands of different sizes. Urology. 2010;76: 1072-6.

9. Robinson D, Van Allen EM, Wu YM, Schultz N, Lonigro RJ, Mosquera JM, et al. Integrative clinical genomics of advanced prostate cancer. Cell. 2015;161:1215-28.

10. Pezaro CJ, et al. Visceral disease in castration-resistant prostate cancer. Eur Urol. 2014;65:270-3.

11. Scher HI, Morris MJ, Stadler WM, Higano C, Basch E, Fizazi K, et al. Prostate Cancer Clinical Trials Working Group 3. Trial design and objectives for castration-resistant prostate cancer: updated recommendations from the Prostate Cancer Clinical Trials Working Group 3. J Clin Oncol. 2016;34(12):1402-18.

12. Ceci F, Herrmann K, Hadaschik B, Castellucci P, Fanti S. Therapy assessment in prostate cancer using choline and PSMA PET/CT. Eur J Nucl Med Mol Imaging. 2017;44(Suppl 1):78-83.

13. Etchebehere E, Brito AE, Rezaee A, Langsteger W, Beheshti M. Therapy assessment of bone metastatic disease in the era of (223)radium. Eur J Nucl Med Mol Imaging. 2017;44(Suppl 1): 84-96.

14. Morigi JJ, Stricker PD, van Leeuwen PJ, Tang R, Ho B, Nguyen Q, et al. Prospective comparison of 18 F-Fluoromethylcholine versus 68Ga-PSMA PET/CT in prostate cancer patients who have rising PSA after curative treatment and are being considered for targeted therapy. J Nucl Med. 2015;56(8):1185-90.

15. Hofman MS, Violet J, Hicks RJ, Ferdinandus J, Thang SP, Akhurst T, et al. [(177)Lu]-PSMA-617 radionuclide treatment in patients with metastatic castration-resistant prostate cancer (LuPSMA trial): a single-centre, single-arm, phase 2 study. Lancet Oncol. 2018;19(6):825-33. 